\title{
Transtornos psiquiátricos menores em usuários de substâncias psicoativas
}

Karine Langmantel Silveira ${ }^{1}$

Michele Mandagará de Oliveira ${ }^{1}$

Poliana Farias Alves ${ }^{1}$

Objetivo: verificar a prevalência de Transtornos Psiquiátricos Menores de acordo com o perfil demográfico e socioeconômico, com o tipo de droga utilizada e com as condições de saúde dos usuários de crack, álcool e outras drogas. Metodologia: estudo transversal, com amostra de 505 usuários. Utilizou-se o Self-Reporting Questionnaire para verificação da prevalência dos transtornos. Resultados: a prevalência encontrada foi de $28,7 \%$, sendo superior entre usuários de crack, mulheres, negros, divorciados ou viúvos, desempregados, entrevistados com problema de saúde, autoavaliação de saúde ruim e que estão insatisfeitos com relacionamentos familiares. Conclusão: constatou-se que a presença dos Transtornos Psiquiátricos Menores pode estar relacionada com alguns fatores que incluem não apenas as características individuas, mas também questões sociais, culturais, econômicas, entre outras.

Descritores: Transtornos Relacionados ao uso de Substâncias; Usuários de Drogas; Transtornos Psiquiátricos Menores. 


\section{Minor psychiatric disorders in users of psychoactive substances}

Objective: To verify the prevalence of Minor Psychiatric Disorders according to the demographic and socioeconomic profile, type of drug used and the health conditions of users of crack, alcohol and other drugs. Methodology: Cross-sectional study, with a sample of 505 users. The Self-Reporting Questionnaire was used to verify the prevalence of disorders. Results: The prevalence found was $28.7 \%$, higher among crack users, women, blacks, divorcees or widowers, unemployed, users with health problems, poor self-assessment of health and who are dissatisfied with family relationships. Conclusions: The presence of Minor Psychiatric Disorders may be related to some factors that include not only individual characteristics, but also social, cultural and economic issues, among others.

Descriptors: Substance-Related Disorders; Drug Users; Minor Psychiatric Disorders.

\section{Trastornos psiquiátricos en usuarios de sustancias psicoativas}

Objetivo: verificar la prevalencia de Trastornos Psiquiátricos Menores de acuerdo con el perfil demográfico y socioeconómico, con el tipo de droga utilizada y con las condiciones de salud de los usuarios de crack, alcohol, y otras drogas. Metodología: estudio transversal, con amuestra de 505 usuarios. Se utilizó el Self-Reporting Questionnaire para verificación de prevalencia de los trastornos. Resultados: la prevalencia encontrada fue de $28,7 \%$, siendo superior entre usuarios de crack, mujeres, negros, divorciados o viudos, desempleados, entrevistados con problemas de salud, mala autoevaluación de salud, y que están insatisfechos con relacionamientos familiares. Conclusión: constatado que la presencia de los trastornos psiquiátricos menores puede estar relacionada con algunos factores que incluyen no solamente las características individuales, pero también cuestiones sociales, culturales, económicas, entre otras.

Descriptores: Trastornos Relacionados con Sustancias; Consumidores de Drogas; Trastornos Psiquiátricos Menores.

\section{Introdução}

Os transtornos psiquiátricos menores (TPM) se referem à situação de saúde de indivíduos que apresentam sintomas de depressão, ansiedade ou sintomas somáticos, entretanto não apresentam critérios formais para o diagnóstico de tais transtornos segundo o DSM-IV (Diagnostic and Statiscal Manual of Mental Disorders - Fourth Edition) e o CID-10 (Classificação Internacional de Doenças $-10^{\mathrm{a}}$ Revisão) ${ }^{(1)}$. Em relação à prevalência desses transtornos mundialmente, estimase que $4,4 \%$ da população global sofre de transtornos depressivos e $3,6 \%$ de transtornos de ansiedade. Essas prevalências variam dependendo da região, sendo encontrada as maiores taxas de depressão na Região Africana $(5,4 \%)$ e de transtornos de ansiedade nas Américas $(5,8 \%)^{(2)}$.

A maioria dos indivíduos com TPM apresenta queixas como tristeza, ansiedade, cansaço, diminuição da concentração, problemas somáticos, irritabilidade e insônia. Essas morbidades menores também podem ser denominadas como transtornos mentais comuns, termo que reforça a frequente ocorrência desses transtornos ${ }^{(3)}$. Esse tipo de queixa é um dos motivos recorrentes na demanda da Atenção Primária à Saúde, contudo, muitas vezes, não recebe a devida atenção e 
tratamento ${ }^{(4)}$. A literatura aponta que os usuários com TPM podem apresentam prejuízos e incapacidades funcionais comparáveis ou mais graves que as pessoas com transtornos crônicos, com impactos econômicos, sociais e sobre o padrão de vida das pessoas e das famílias ${ }^{(5)}$.

Para a detecção dos TPM, criou-se, em 1980, o Self-Reporting Questionnaire (SRQ-20), o qual sugere nível de suspeição (presença/ausência) de algum transtorno mental. O mesmo avalia se há algum transtorno, mas não oferece especificidade do tipo de transtorno existente. Por esse caráter de triagem, esse questionário é bastante adequado para estudos de populações, sendo muito útil para uma primeira classificação de possíveis casos e não casos ${ }^{(6)}$.

Segundo a OMS, o contexto social de pessoas e grupos pode influenciar significativamente no surgimento de problemas de saúde mental, tais como TPM. Famílias vivendo na pobreza, grupos minoritários, pessoas com problemas de saúde crônicos, pessoas que sofrem discriminação e violações dos direitos humanos, crianças negligenciadas e pessoas expostas ao abuso de Substâncias Psicoativas (SPA) se apresentam como principais grupos vulneráveis ${ }^{(7)}$.

De modo mais desafiador se apresenta o TPM associado ao consumo abusivo de SPA. Essa comorbidade pode ser um fator agravante, indutor $\mathrm{e}$ perpetuador da condição de dependência ou abuso da substância, bem como pode interferir na qualidade de vida do usuário de substâncias psicoativas, inferindo, assim, uma maior necessidade de investigação desse fenômeno ${ }^{(8-9)}$.

Dessa forma, este estudo teve por objetivo verificar a prevalência de Transtornos Psiquiátricos Menores de acordo com o perfil demográfico e socioeconômico, com o tipo de substância psicoativa utilizada e com as condições de saúde dos usuários de crack, álcool e outras drogas do município de Pelotas-RS.

\section{Metodologia}

Trata-se de um estudo transversal e exploratório que é parte integrativa da pesquisa "Perfil dos Usuários de Crack e Padrões de Uso", financiada pelo Conselho Nacional de Desenvolvimento Científico e Tecnológico (CNPq), edital MCT/CNPq n ${ }^{\circ}$ 041/2010.

A mesma foi desenvolvida em um município do interior do estado do Rio Grande do Sul, o qual conta com dois serviços distintos voltados à atenção psicossocial de pessoas usuárias de crack, álcool e outras drogas. A prevalência de usuários de drogas foi desconhecida $(p=0,50)$, admitiu-se um erro amostral de $4 \%(d=0,04)$, sob o nível de confiança de $95 \%$ $(\alpha=0,05)$, e o número de participantes em cada estrato foi proporcional ao total de usuários cadastrados. A amostra final foi constituída por 505 usuários. A sistemática de seleção dos entrevistados adotada foi o sorteio nominal direto nas bases de dados dos dois serviços.

Para o presente estudo, selecionaram-se as seguintes variáveis: variável dependente o Escore do $S R Q$ adaptado e como variáveis independentes o perfil demográfico e socioeconômico, o tipo de droga que o entrevistado já utilizou na vida, se apresenta algum problema de saúde, autoavaliação de saúde e relacionamento com a família.

O SRQ é um questionário que contribui com a identificação de distúrbios psiquiátricos na atenção básica. Ele foi criado em quatro países em desenvolvimento (Colômbia, Sudão, Índia e Filipinas) e validado no Brasil em 1986 ${ }^{(10)}$. É composto por 20 questões elaboradas para deteç̧ão de distúrbios "neuróticos". Para uma pessoa ser considerada como possível caso, utiliza-se a pontuação de oito ou mais respostas afirmativas na subescala de sintomas "neuróticos" para mulheres e seis ou mais respostas afirmativas para homens. Esse ponto foi obtido anteriormente através de determinação da sensibilidade, especificidade e dos valores preditivos positivos e negativos em outras amostras ${ }^{(11)}$.

Inicialmente, durante o teste piloto, utilizou-se a escala SRQ com 20 questões, porém, para facilitar a compreensão dos usuários, os participantes solicitaram que fossem agrupadas quatro questões em duas, visto que elas se tratavam de assunto semelhante, assim utilizou-se o SRQ com 18 questões. Visando validar a consistência interna das respostas, aplicou-se o Coeficiente Alfa de Cronbach, no qual os resultados do presente estudo mantiveram-se dentro do limite 0,7 a 0,9 representando uma boa consistência interna ${ }^{(12)}$, conforme pode-se observar na Tabela 1.

Os questionários aplicados foram codificados pelo entrevistador e revisados pelos coordenadores. Os dados foram digitados através do gerenciador de banco de dados Microsoft Access v.2003.

Realizou-se a análise dos dados utilizando o software STATA v.12, que consistiu na descrição das frequências absolutas e relativas das características demográficas e socioeconômicas de toda a amostra e estratificadas de acordo com a classificação do Escore SRQ (positivo ou negativo). Utilizaram-se distribuições de frequências bivariadas e medidas descritivas (médias e desvio padrão). As análises bivariadas foram realizadas com o objetivo de descrever e verificar diferenças proporcionais entre os grupos de estratificação através dos Testes Chi-quadrado de Pearson para as variáveis nominais e 
o Qui-quadrado de Tendência Linear para as variáveis ordinais. Aplicou-se o teste T de Student para verificar diferenças entre o escore SRQ médio dos usuários (de crack e outras drogas). O nível de significância adotado em todos os testes foi de $5 \%$ ( $p \leq 0,05)$.

A pesquisa obedeceu aos princípios éticos da Resolução COFEN n 311/2007 e resoluções 196/96 e 466/12 do Conselho Nacional de Saúde do Ministério da Saúde. O projeto foi aprovado pelo Comitê de Ética e Pesquisa da Faculdade de Enfermagem da Universidade Federal de Pelotas de Pelotas recebendo o parecer $n^{\circ} 301 / 2011$.

Tabela 1. Alfa de Cronbach do SRQ -18 ( $n=505)$. Pelotas - RS, Brasil, 2012

\begin{tabular}{|c|c|}
\hline Item & $\begin{array}{c}\text { Alfa de } \\
\text { Cronbach* }\end{array}$ \\
\hline $\begin{array}{l}\text { Sr(a). tem dores de cabeça com } \\
\text { frequência? }\end{array}$ & 0,82 \\
\hline Tem falta de apetite? & 0,82 \\
\hline $\mathrm{O}(\mathrm{a}) \mathrm{Sr}(\mathrm{a})$. dorme mal? & 0,82 \\
\hline $\mathrm{O}(\mathrm{a}) \mathrm{Sr}(\mathrm{a})$. fica com medo com facilidade? & 0,83 \\
\hline Suas mãos tremem? & 0,82 \\
\hline $\begin{array}{l}\text { O(a) } \operatorname{Sr}(a) \text {. se sente nervoso }(a) \text {, tenso(a) } \\
\text { ou preocupado(a)? }\end{array}$ & 0,82 \\
\hline $\begin{array}{l}\text { Sua digestão não é boa ou sofre de } \\
\text { perturbação digestiva? }\end{array}$ & 0,83 \\
\hline $\begin{array}{l}\text { O(a) } \operatorname{Sr}(a) \text {. não consegue pensar com } \\
\text { clareza? }\end{array}$ & 0,83 \\
\hline Sente-se infeliz? & 0,82 \\
\hline $\mathrm{O}(\mathrm{a}) \mathrm{Sr}(\mathrm{a})$. chora mais que o comum? & 0,82 \\
\hline $\begin{array}{l}\text { Acha difícil apreciar (gostar de) suas } \\
\text { atividades diárias? }\end{array}$ & 0,82 \\
\hline Acha difícil tomar decisões? & 0,82 \\
\hline $\begin{array}{l}\text { Seu trabalho diário é um sofrimento? } \\
\text { Tormento? Tem dificuldade em fazer seu } \\
\text { trabalho? }\end{array}$ & 0,83 \\
\hline $\begin{array}{l}\text { O(a) } \operatorname{Sr}(a) \text {. não é capaz de ter um papel útil } \\
\text { na vida? }\end{array}$ & 0,84 \\
\hline $\mathrm{O}(\mathrm{a}) \mathrm{Sr}(\mathrm{a})$. perdeu interesse nas coisas? & 0,82 \\
\hline $\begin{array}{l}\text { Acha que é uma pessoa que não vale } \\
\text { nada? }\end{array}$ & 0,82 \\
\hline $\begin{array}{l}\text { O pensamento de acabar com a sua vida já } \\
\text { passou por sua cabeça? }\end{array}$ & 0,82 \\
\hline $\begin{array}{l}\text { O(a) } \mathrm{Sr}(a) \text {. se sente cansado(a) todo o } \\
\text { tempo? }\end{array}$ & 0,82 \\
\hline \multicolumn{2}{|c|}{$\begin{array}{l}\text { *Alfa de Cronbach com a retirada dos itens do domínio; Alfa de } \\
\text { Cronbach global }=0,83 \text {. }\end{array}$} \\
\hline
\end{tabular}

\section{Resultados}

A população do estudo constitui-se por 505 usuários de SPA, o perfil demográfico dos entrevistados foi predominante do sexo masculino $(83,8 \%)$, cor da pele autorreferida branca $(50,9 \%)$, mais da metade dos entrevistados eram solteiros (53,3\%). A média de idade foi de $38 \pm 13,1$ anos, com mínima de 18 e máxima de 76 anos. Já o perfil socioeconômico revelou indivíduos com baixo nível de escolaridade, em que $77,8 \%$ possuíam apenas o ensino fundamental completo ou incompleto. No que tange ao rendimento familiar, $26 \%$ não possuíam renda ou a renda era inferior a 1 salário mínimo (sm). Quanto à situação ocupacional, 29,1\% estavam desempregados e apenas $23,4 \%$ possuíam vínculo formal de emprego.

Em relação à prevalência de TPM na amostra, observou-se que $28,7 \%$ dos entrevistados obtiveram rastreio positivo na escala SRQ. Na Tabela 2, estão descritas as características demográficas e socioeconômicas dos usuários de drogas e estratificadas segundo o escore SRQ.

A relação entre o escore do $S R Q$ e a idade revelase direta, observando-se aumento da frequência de indivíduos com rastreio positivo para TPM com idade entre 30 e 39 anos. No entanto, essas tendências proporcionais não se mostraram estatisticamente significantes entre os grupos.

Identificaram-se diferenças proporcionais estatisticamente significantes entre os grupos com relação à cor $(p=0,029)$. A população do estudo foi predominantemente branca, contudo a relação entre cor da pele e o escore $S R Q$ revela predomínio de usuários negros com TPM nesse grupo.

Também se verificaram diferenças proporcionais estatisticamente significantes entre o escore SRQ e a situação conjugal $(p=0,008)$. Observou-se que a maior prevalência de TPM se concentrou no grupo de pessoas que declararam divorciados/viúvos/ separados.

Não foram verificadas tendências proporcionais estatisticamente significantes entre os grupos e a escolaridade.

Verificaram-se tendências proporcionais estatisticamente significantes entre a situação de atividade econômica e o escore SRQ $(p=0,02)$, sendo observada maior prevalência de TPM entre usuários que disseram não trabalharem.

Apesar do predomínio do sexo masculino entre os usuários entrevistados (83,8\%), a prevalência de TPM foi superior entre as mulheres $(32,9 \%)$, merecendo destaque na análise.

Na Figura 1, apresenta-se a distribuição do escore SRQ com estratificação por sexo e tipo de SPA utilizada.

Do total de 505 usuários entrevistados, os usuários de drogas (exceto o crack) foram o grupo predominante, com 369 entrevistados $(73,1 \%)$, se comparados com 
os entrevistados que relataram fazer uso do crack, 136 usuários (26,9\%). Todavia, pode-se observar na Figura 1 que o Escore do SRQ foi maior entre os usuários de crack. O sexo feminino apresentou em ambos os grupos maior dispersão e maior concentração de valores superiores ao valor mediano da amostra $(\mathrm{md}=3$, média=4,1 e desvio padrão=3,7). O sexo masculino apresentou menor valor mediano do escore SRQ em ambos os grupos.

Em relação aos tipos de SPA já experimentado pelo menos uma vez na vida pelos usuários, as mais frequentemente citadas em ordem decrescente de resposta foram o álcool $(96,1 \%)$, o tabaco $(81,4 \%)$, a maconha $(52,4 \%)$, a cocaína $(40,2 \%)$ e o crack $(26,7 \%)$. A

Tabela 2. Distribuição do Escore SRQ segundo características demográficas e socioeconômicas dos usuários de crack, álcool e outras drogas $(n=505)$. Pelotas - RS, Brasil, 2012

\begin{tabular}{|c|c|c|c|c|}
\hline \multirow[b]{2}{*}{ Característica } & \multirow{2}{*}{$\begin{array}{l}\text { Total } \\
\text { n (\%) }\end{array}$} & \multicolumn{2}{|c|}{ Escore SRQ } & \multirow[b]{2}{*}{ p-valor } \\
\hline & & $\begin{array}{c}\text { Negativo } \\
\mathrm{n}(\%)\end{array}$ & $\begin{array}{c}\text { Positivo } \\
\mathrm{n}(\%)\end{array}$ & \\
\hline \multicolumn{5}{|l|}{ Sexo } \\
\hline Feminino & $82(16,2)$ & $55(67,1)$ & $27(32,9)$ & $0,357^{*}$ \\
\hline Masculino & $423(83,8)$ & $305(72,1)$ & $118(27,9)$ & \\
\hline \multicolumn{5}{|l|}{ Grupo etário } \\
\hline$<20$ & $18(3,6)$ & $16(88,9)$ & $2(11,1)$ & $0,17^{\dagger}$ \\
\hline 20 a 24 & $65(12,9)$ & $51(78,5)$ & $14(21,5)$ & \\
\hline 25 a 29 & $66(13,1)$ & $45(68,2)$ & $21(31,8)$ & \\
\hline 30 a 39 & $133(26,4)$ & $94(70,7)$ & $39(29,3)$ & \\
\hline 40 a 49 & $110(21,8)$ & $76(69,1)$ & $34(30,9)$ & \\
\hline 50 a 59 & $84(16,6)$ & $56(66,7)$ & $28(33,3)$ & \\
\hline 60 ou mais & $29(5,7)$ & $22(75,9)$ & $7(24,1)$ & \\
\hline \multicolumn{5}{|l|}{ Cor da pele } \\
\hline Branca & $257(50,9)$ & $181(70,4)$ & $76(29,6)$ & $0,029^{*}$ \\
\hline Parda/mestiça & $98(19,4)$ & $76(77,6)$ & $22(22,4)$ & \\
\hline Preta & $111(22,0)$ & $70(63,2)$ & $41(36,9)$ & \\
\hline Outra & $39(7,7)$ & $33(84,6)$ & $6(15,4)$ & \\
\hline \multicolumn{5}{|l|}{ Situação conjugal } \\
\hline Casado/com companheiro & $170(33,7)$ & $130(76,5)$ & $40(23,4)$ & $0,008^{*}$ \\
\hline Solteiro & $269(53,3)$ & $191(71,0)$ & $78(29,0)$ & \\
\hline Divorciado/viúvo/separado & $66(13,0)$ & $39(59,1)$ & $27(40,9)$ & \\
\hline \multicolumn{5}{|l|}{ Escolaridade } \\
\hline Sem escolaridade & $10(2,0)$ & $8(80,0)$ & $2(20,0)$ & $0,07^{\dagger}$ \\
\hline Fund. incompleto/completo & $393(77,8)$ & $271(69,0)$ & $122(31,0)$ & \\
\hline Médio incompleto/completo & $88(17,4)$ & $68(77,3)$ & $20(22,7)$ & \\
\hline Superior incompleto/completo & $14(2,8)$ & $13(92,9)$ & $1(7,1)$ & \\
\hline \multicolumn{5}{|l|}{ Situação de Atividade Econômica } \\
\hline Não & $147(29,1)$ & $92(62,6)$ & $55(37,4)$ & $0,02^{+}$ \\
\hline Trabalho informal & $106(21,0)$ & $77(72,6)$ & $29(27,4)$ & \\
\hline Trabalho formal & $118(23,3)$ & $92(78,0)$ & $26(22,0)$ & \\
\hline Autônomo & $123(24,4)$ & $91(74,0)$ & $32(26,0)$ & \\
\hline Eventual & $11(2,2)$ & $8(72,7)$ & $3(27,3)$ & \\
\hline \multicolumn{5}{|l|}{ Renda Familiar ${ }^{\ddagger}$} \\
\hline Sem renda & $19(3,8)$ & $12(63,2)$ & $7(36,8)$ & $0,07^{\dagger}$ \\
\hline < 1 salário & $112(22,2)$ & $76(67,9)$ & $36(32,1)$ & \\
\hline 1 até 2 salários & $199(39,4)$ & $137(68,8)$ & $62(32,3)$ & \\
\hline Superior a 2 até 4 salários & $114(22,6)$ & $90(79,0)$ & $24(21,0)$ & \\
\hline Mais de 4 salários & $48(9,5)$ & $40(83,3)$ & $8(16,7)$ & \\
\hline Não soube/não informou & $13(2,6)$ & $5(38,5)$ & $8(61,5)$ & \\
\hline
\end{tabular}

Fonte: Projeto de pesquisa "Perfil dos usuários de crack e padrões de uso - Pelotas 2014"(13).

*Teste Qui-quadrado de Pearson; †Teste Qui-quadrado de Tendência Linear; ¥Salário mínimo na época R\$ 622,00. 


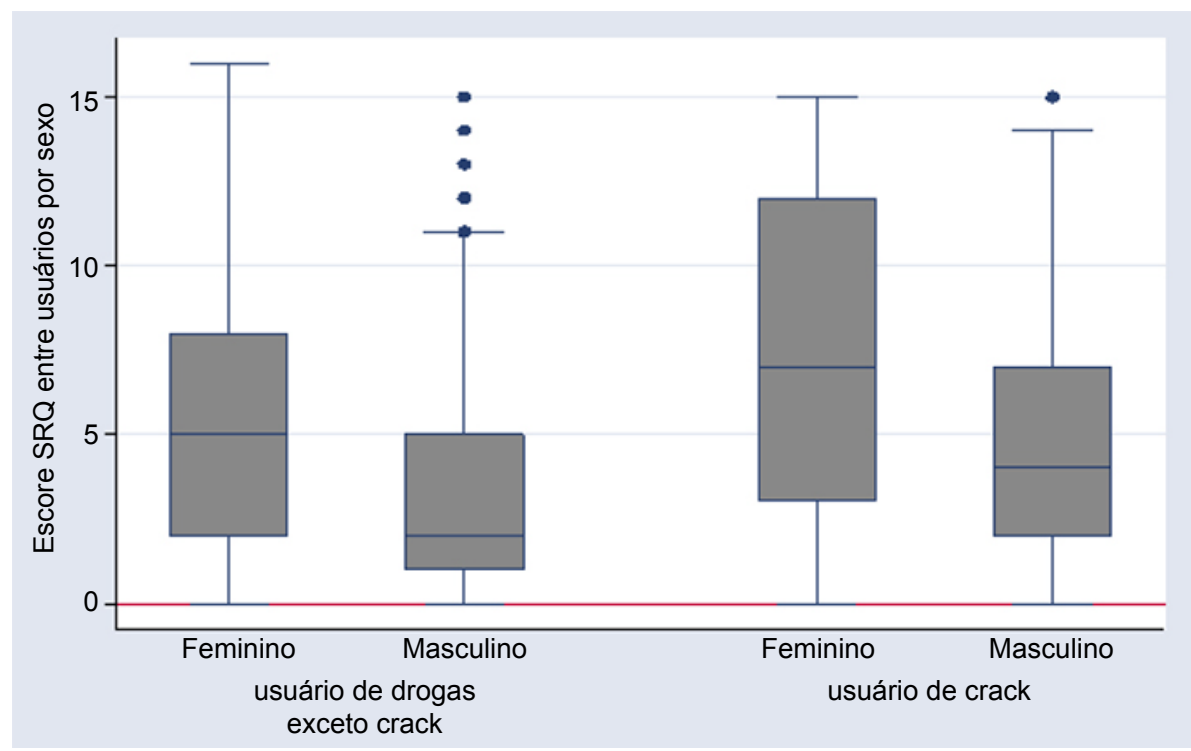

Fonte: Projeto de pesquisa "Perfil dos usuários de crack e padrões de uso - Pelotas 2014"(13)

Figura 1. Distribuição do escore $S R Q$ entre usuários por sexo $(n=505)$. Pelotas - RS, 2012

Tabela 3. Distribuição do Escore $S R Q$ segundo drogas que o entrevistado experimentou $(n=505)$. Pelotas $-R S$, Brasil, 2012

\begin{tabular}{|c|c|c|c|c|}
\hline \multirow[b]{2}{*}{ Característica } & \multirow{2}{*}{$\begin{array}{c}\text { Total } \\
\mathrm{n}^{*}(\%)\end{array}$} & \multicolumn{2}{|c|}{ Escore SRQ } & \multirow[b]{2}{*}{ p-valor ${ }^{\dagger}$} \\
\hline & & $\begin{array}{c}\text { Negativo } \\
\mathrm{n}(\%)\end{array}$ & $\begin{array}{c}\text { Positivo } \\
\text { n (\%) }\end{array}$ & \\
\hline \multicolumn{5}{|l|}{ Álcool } \\
\hline Não & $19(3,9)$ & $12(63,2)$ & $7(36,8)$ & 0,432 \\
\hline Sim & $477(96,1)$ & $341(71,5)$ & $136(28,5)$ & \\
\hline \multicolumn{5}{|l|}{ Tabaco } \\
\hline Não & $92(18,6)$ & $71(77,2)$ & $21(22,8)$ & 0,164 \\
\hline Sim & $402(81,4)$ & $281(69,9)$ & $121(30,1)$ & \\
\hline \multicolumn{5}{|l|}{ Crack } \\
\hline Não & $370(73,3)$ & $283(76,5)$ & $87(23,5)$ & 0,000 \\
\hline Sim & $135(26,7)$ & $77(57,0)$ & $58(43,0)$ & \\
\hline \multicolumn{5}{|l|}{ Cocaína } \\
\hline Não & $296(59,8)$ & $228(77,0)$ & $68(23,0)$ & 0,000 \\
\hline Sim & $199(40,2)$ & $124(62,3)$ & $75(37,7)$ & \\
\hline \multicolumn{5}{|l|}{ Maconha } \\
\hline Não & $233(47,6)$ & $170(73,0)$ & $63(27,0)$ & 0,353 \\
\hline Sim & $256(52,4)$ & $177(69,1)$ & $79(30,9)$ & \\
\hline \multicolumn{5}{|l|}{ Substâncias sintéticas } \\
\hline Não & $449(91,8)$ & $321(71,5)$ & $128(28,5)$ & 0,386 \\
\hline Sim & $40(8,2)$ & $26(65,0)$ & $14(35,0)$ & \\
\hline
\end{tabular}

Fonte: Projeto de pesquisa "Perfil dos usuários de crack e padrões de uso - 2014"(13).

"número variável devido à possibilidade de múltipla resposta e usuários que não informaram; †Teste Qui-quadrado de Pearson.

distribuição do escore do SRQ segundo essas substâncias utilizadas foram descritas na Tabela 3.

Ao se verificar a associação entre as drogas utilizadas e o escore $S R Q$, constatou-se que os usuários que referiram ter experimentado crack pelo menos uma vez na vida apresentaram uma prevalência de TPM quase duas vezes superior ao da população de estudo $(28,7 \%$ vs $43 \%)$, seguida do uso de cocaína ( $28,7 \%$ vs $37,7 \%$ ). Os usuários que referiram experimentar esses dois tipos de SPA apresentaram diferenças proporcionais estatisticamente significantes quanto ao escore $S R Q(p<0,05)$.

Após investigar a variância do escore $S R Q$ entre os usuários (de crack e de outras drogas), verificou-se através do teste T de Student que há evidências estatisticamente significantes de que o valor médio do escore $S R Q$ difere entre os usuários $(p<0,001)$. 
Na Tabela 4, tem-se a associação entre o escore SRQ e as características relacionadas à situação de saúde e ao relacionamento com a família entre usuários.

Quanto à situação de saúde dos usuários, nota-se uma prevalência de TPM superior entre os que referiram ter problemas de saúde (42,9\%), dado este no qual se verificam diferenças proporcionais estatisticamente significantes $(p<0,001)$.

Identificou-se uma relação direta com tendências proporcionais estatisticamente significantes no padrão de respostas da percepção de saúde e o aumento da prevalência de TPM, no qual quem tem a pior avaliação de saúde apresentou maior prevalência $(p<0,001)$.

A relação com familiares e o escore $S R Q$ revela que usuários insatisfeitos com suas relações apresentam prevalência mais de TPM se comparados com os que estão satisfeitos, sendo esse resultado estatisticamente significante $(p=0,009)$.

Tabela 4. Distribuição do Escore $S R Q$ segundo situação de saúde e relacionamento com a família $(n=505)$. Pelotas - RS, Brasil, 2012

\begin{tabular}{|c|c|c|c|c|}
\hline \multirow[b]{2}{*}{ Característica } & \multirow{2}{*}{$\begin{array}{l}\text { Total } \\
\text { n (\%) }\end{array}$} & \multicolumn{2}{|c|}{ Escore SRQ } & \multirow[b]{2}{*}{ p-valor } \\
\hline & & $\begin{array}{c}\text { Negativo } \\
\mathrm{n}(\%)\end{array}$ & $\begin{array}{c}\text { Positivo } \\
\text { n (\%) }\end{array}$ & \\
\hline \multicolumn{5}{|l|}{ Problemas de saúde } \\
\hline Não & $323(64,0)$ & $256(79,3)$ & $67(20,7)$ & $0,000^{*}$ \\
\hline $\operatorname{Sim}$ & $182(36,0)$ & $104(57,1)$ & $78(42,9)$ & \\
\hline \multicolumn{5}{|l|}{ Autoavaliação da saúde } \\
\hline Muito bom & $107(21,2)$ & $92(86,0)$ & $15(14,0)$ & $0,00^{+}$ \\
\hline Bom & $258(51,1)$ & $200(77,5)$ & $58(22,5)$ & \\
\hline Regular & $111(22,0)$ & $56(50,5)$ & $55(49,5)$ & \\
\hline Mal & $24(4,7)$ & $10(41,7)$ & $14(58,3)$ & \\
\hline Muito mal & $5(1,0)$ & $2(40,0)$ & $3(60,0)$ & \\
\hline \multicolumn{5}{|l|}{ Relacionamento com a família } \\
\hline Insatisfeito & $124(24,6)$ & $75(60,5)$ & $49(39,5)$ & $0,009^{*}$ \\
\hline Satisfeito & $378(74,8)$ & $283(74,9)$ & $95(25,1)$ & \\
\hline Não respondeu & $3(0,6)$ & $2(66,7)$ & $1(33,3)$ & \\
\hline
\end{tabular}

Fonte: Projeto de pesquisa "Perfil dos usuários de crack e padrões de uso - 2014" (13).

*Teste Qui-quadrado de Pearson; †Teste Qui-quadrado de Tendência Linear.

\section{Discussão}

Pesquisas apontam que os TPM podem acometer pessoas de todas as regiões do mundo, contudo, constatou-se que a prevalência desses transtornos é maior em grupos populacionais específicos, e entre eles encontram-se os usuários abusivos de SPA ${ }^{(2,7,14-16)}$.

O presente estudo revelou prevalência de TPM nos usuários de SPA de $28,7 \%$, resultado este menor do que o observado em estudo realizado com usuários de SPA no estado de Goiás $(37,3 \%)^{(15)}$. Contudo, quando são observados apenas os usuários que referiram ter experimentado crack pelo menos uma vez na vida, estes apresentaram uma propensão de desenvolvimento de TPM maior do que os demais usuários de SPA. Resultado este que vai ao encontro da literatura quando aponta que os usuários de crack apresentam maior ocorrência de sintomas depressivos e ansiosos do que os usuários de outras substâncias ${ }^{(17)}$.
A ocorrência dos TPM pode estar relacionada com alguns determinantes preditos do surgimento dos distúrbios de saúde mental que incluem não apenas as características individuais, mas também fatores sociais, culturais, econômicos, políticos e ambientais $^{(2)}$. Destacando, assim, que a vulnerabilidade biopsicossocial em que a maioria dos usuários de SPA se encontra poderia justificar maiores prevalências.

Em relação às características individuais que apresentaram relação estatisticamente significativa com o rastreio positivo para os TPM, nesta pesquisa, observou-se a cor da pele autorreferida preta $(36,9 \%)$, ser divorciado, viúvo ou separado $(40,9 \%)$ e não estar trabalhando no momento em que a entrevista ocorreu $(37,4 \%)$. Já a variável sexo, mesmo não dando diferenças estatisticamente significantes, merece destaque, visto que mesmo a maioria dos entrevistados sendo do sexo masculino, a prevalência de TPM foi superior entre as mulheres $(32,8 \%)$. Resultados estes que estão em consonância com a literatura, na qual se observou 
maior prevalência dos transtornos na maioria desses grupos tanto em estudos com usuários de SPA como em estudos de base populacional(4,15,17-18).

Constatou-se também a influência da situação de saúde e relacionamento familiar na variação da prevalência dos TPM. Os participantes que relataram apresentar problema de saúde, autoavaliaram sua saúde como muito ruim e esboçaram estar insatisfeitos com o relacionamento familiar foram os que apresentaram as maiores prevalências de TPM se comparados com os que relataram não ter problema de saúde, os que autoavaliaram sua saúde como boa, muito boa ou regular e os que esboçaram estar satisfeitos com o relacionamento familiar.

A partir desses resultados, pode-se inferir que a presença de sintomas psíquicos como o de ansiedade e depressão associada ao abuso de SPA é frequente, ressaltando que a comorbidade TPM poderia aumentar a gravidade dos sintomas relacionados ao abuso de SPA, bem como ter influência em diversos âmbitos da vida desse usuário, tornando, assim, necessária a sua correta identificação.

Entretanto, por se tratar de um estudo transversal, não pode ser confirmado se a utilização de SPA acarreta na presença de TPM ou se é a presença desses transtornos que influencia na utilização da substância. De qualquer modo, é sabido que um contexto social de vulnerabilidade pode interferir tanto no surgimento de TPM como na utilização de SPA. Dessa forma, salienta-se a necessidade de maiores aprofundamentos acerca dos fatores sociais de vulnerabilidade, de forma a se pensar e agir de modo mais contundente para a diminuição das inequidades sociais. De modo clarividente, um trabalho focalizado nesse objetivo poderia de forma efetiva elevar o cuidado em saúde baseado apenas nos aspectos biológicos para um patamar mais elevado, de um cuidado biopsicossocial.

\section{Conclusão}

Com a realização deste estudo, constatou-se que a presença dos Transtornos Psiquiátricos Menores pode estar relacionada com alguns fatores que incluem não apenas as características individuais, mas também questões sociais, culturais, econômicas, entre outras. Portanto, enfatiza-se a necessidade de uma rede de atenção aos usuários de SPA na qual os profissionais de saúde estejam atentos para o outro, tentando identificar seus anseios e necessidades, e para isso o olhar do profissional deve ir além do motivo que fizeram os usuários procurarem o serviço de saúde. Ao adotar essa conduta no momento assistencial, o profissional assume que a saúde e a doença não são apenas objetos, e sim, configuram-se como o modo de ser e andar na vida.

\section{Referências}

1. Tavares JP, Beck CLC, Magnago TSBS, Greco PBT, Prestes FC, Silva RM. Scientific production on the minor psychological distress from the Self-report questionnaire. Rev Enferm UFSM. 2011; 1(1):113-23.

2. World Health Organization. Depression and Other Common Mental Disorders: Global Health Estimates. Geneva: WHO; 2017.

3. Cachoeira DVAC, Santos SCC, Meneganti APS, Negreiros NF, Cardoso L, Preto VA. Relation of sociodemographic profile with the risk of illness by mental disorders common among students of the nursing course. Rev Enferm UFPE on line. 2016; 10(12):4501-8.

4. Gama CAP, Campos RTO, Ferrer AL. Saúde mental e vulnerabilidade social: a direção do tratamento. Rev Latinoam Psicopatol Fundam. 2014;17(1):69-84.

5. Borges TL, Hegadoren KM, Miasso DI. Transtornos mentais comuns e uso de psicofármacos em mulheres atendidas em unidades básicas de saúde em um centro urbano brasileiro. Rev Panam Salud Publica. 2015 sep;38(3):195-201.

6. Santos KOB, Araujo TM, Pinho PS, Silva ACC. Avaliação de um instrumento de mensuração de morbidade psíquica: estudo de validação do self-reporting questionnaire (srq-20). Rev Baiana Saúde Pública. 2010 jul./set; 34(3):544-60.

7. World Health Organization. Mental Health: Action Plan 2013-2020. Geneva: WHO; 2013.

8. Kluwe-Schiavon B, Tractenberg SG, Sanvicente-Vieira B, Rosa CSO, Arteche AX, Pezzi JC, et al. Propriedades psicométricas da Cocaine Selective Severity Assessment (CSSA) em mulheres usuárias de crack. J Bras Psiquiatr. 2015; 64(2):115-21.

9. Grossi FT, Oliveira RM. Manejo clínico do usuário de crack. Minas Gerais: Diretrizes clínicas; 2013.

10. Harding TW, Arango MV, Baltazar J, Climent CE, Ibrahim HHA, Ladrido-Ignacio L. Mental disorders in primary health care: a study of their frequency and diagnosis in four developing countries. Psychol Med. 1980; 10:231-41. 11. Mari JJ, Williams P. A validity study of a psychiatric screening questionnaire (SRQ-20) in primary care in the city of São Paulo. Br J Psychiatry. 1986; 148:23-6.

12. Bruni AL. SPSS aplicado à pesquisa acadêmica. São Paulo: Atlas; 2009

13. Oliveira MM, Coimbra VCC. Relatório Final: Perfil dos Usuários de Crack e Padrões de Uso. Pelotas (RS): Faculdade de Enfermagem da Universidade Federal de Pelotas. 2014; 85p. 
14. Formigoni, MLOS, organizadora. Atenção integral na rede de saúde: módulo 5. - 11. e 12. ed. Brasília: Secretaria Nacional de Políticas sobre Drogas; 2017.

15. Lucchese R, Silva PCD, Denardi TC, Felipe RL, Vera I, Castro PA, Bueno AA, Fernandes IL. Common mental disorder among alcohol and drug abusers: a cross-sectional study. Texto Contexto Enferm, 2017; 26(1):e4480015.

16. Merchán-Hamann E, Leal EM, Basso ML, García EM, Reid P, Kulakova OV, et al. Comorbilidad entre abuso/ dependencia de drogas y el distrés psicológico en siete países de Latinoamérica y uno del Caribe. Texto Contexto Enferm. 2012; 21(espp): 87-95.

17. Horta RL, Horta BL, Rosset AP, Horta CL. Perfil dos usuários de crack que buscam atendimento em Centros de Atenção Psicossocial. Cad Saúde Pública. 2011 nov; 27(11):2263-70.

18. Gonçalves DA, Mari JJ, Bower P, Gask L, Dowrick C, Tófoli LF et al. Brazilian multicentre study of common mental disorders in primary care: rates and related social and demographic factors. Cad Saúde Pública. 2014; 30(3):623-32. 